\title{
Half-integral weight $p$-adic coupling of weakly holomorphic and holomorphic modular forms
}

\author{
Kathrin Bringmann ${ }^{1 *}$, Pavel Guerzhoy ${ }^{2}$ and Ben Kane ${ }^{3 *}$ (1)
}

\author{
*Correspondence: \\ kbringma@math.uni-koeln.de; \\ ben.kane@gmail.com \\ 1 Mathematical Institute, University \\ of Cologne, Weyertal 86-90, 50931 \\ Cologne, Germany \\ 3 Department of Mathematics, \\ University of Hong Kong, Pokfulam, \\ Hong Kong \\ Full list of author information is \\ available at the end of the article
}

\begin{abstract}
In this paper, we consider $p$-adic limits of $\beta^{-n} g \mid U_{p^{2}}^{n}$ for half-integral weight weakly holomorphic Hecke eigenforms $g$ with eigenvalue $\lambda_{p}=\beta+\beta^{\prime}$ under $T_{p^{2}}$ and prove that these equal classical Hecke eigenforms of the same weight. This result parallels the integral weight case, but requires a much more careful investigation due to a more complicated structure of half-integral weight weakly holomorphic Hecke eigenforms.
\end{abstract}

Keywords: Weakly holomorphic modular forms; $p$-adic congruences; Half-integral weight; Hecke eigenforms; Shimura lift; Shintani lift

\section{Introduction and statement of the results}

In this paper, we establish a $p$-adic relation between half-integral weight weakly holomorphic modular forms and classical half-integral weight holomorphic modular forms. Roughly speaking, we show that the $p^{2 n} m$ th coefficient of a weakly holomorphic Hecke eigenform with algebraic coefficients is congruent to the $m$ th coefficient of a holomorphic Hecke eigenform modulo a high power of $p$. A similar relation for integral weight modular forms was recently proven in [11] and was later shown by the authors [3] to be related to the occurrence of a $p$-adic modular form. The similarity between the integral and halfintegral weight cases is far from being obvious. The $p$-adic relations proven in [11] rely heavily on the construction of integral weight weakly holomorphic Hecke eigenforms. The construction of half-integral weight weakly holomorphic Hecke eigenforms is much more delicate. The fact that the resulting $p$-adic relations parallel those in the integral weight case strongly supports the definition of half-integral weight weakly holomorphic Hecke eigenforms found in [4].

In order to define weakly holomorphic Hecke eigenforms, we first require some notation. Throughout, $\kappa$ denotes an even integer or a half-integer. If $\kappa$ is an even integer, we let $M_{\kappa}^{!}$be the infinite-dimensional space of weight $\kappa$ weakly holomorphic modular forms on $\mathrm{SL}_{2}(\mathbb{Z})$, i.e., meromorphic modular forms whose only possible poles lie at the cusps. Similarly, if $\kappa$ is a half-integer, we write $\mathbb{M}_{\kappa}^{!}$for the space of weakly holomorphic modular forms of weight $\kappa$ on $\Gamma_{0}(4)$ which lie in Kohnen's plus space [12]. After subtracting a constant multiple of the Eisenstein series, one obtains an element of the space $S_{\kappa}^{!}\left(\mathbb{S}_{\kappa}^{!}\right.$if $\kappa$ is half-integral) of weakly holomorphic cusp forms, i.e., those weakly holomorphic modular

(c) 2015 Bringmann et al. Open Access This article is distributed under the terms of the Creative Commons Attribution 4.0 International License (http://creativecommons.org/licenses/by/4.0/), which permits unrestricted use, distribution, and reproduction in any medium, provided you give appropriate credit to the original author(s) and the source, provide a link to the Creative Commons license, and indicate if changes were made. 
forms with vanishing constant coefficient. We denote the subspaces of holomorphic cusp forms by $S_{\kappa} \subset S_{\kappa}^{!}$(resp. $\mathbb{S}_{\kappa} \subset \mathbb{S}_{\kappa}^{!}$).

There is a natural action on each of the above spaces by the Hecke operators $T_{p^{v}}$, where $p$ is a prime and $v=1$ (resp. $v=2$ and $p>2$ ) if the weight is integral (resp. halfintegral). However, the naive definition of a Hecke eigenform fails in general for weakly holomorphic modular forms since the orders of the poles at cusps increase when applying $T_{p^{v}}$. To remedy this problem in integral weight, Hecke eigenforms in a natural quotient space were instead considered in [10]. We call a weakly holomorphic cusp form $f$ of weight $\kappa$ a weakly holomorphic Hecke eigenform if for each prime $p$ there exists $\lambda_{p} \in \mathbb{C}$ such that

$$
f \mid T_{p^{v}}-\lambda_{p} f \in J_{\kappa} .
$$

Here, for $\kappa \in \mathbb{N}$, the Hecke-stable subspaces $J_{\kappa} \subset S_{\kappa}^{!}$are defined by

$$
J_{\kappa}:=D^{\kappa-1}\left(S_{2-\kappa}^{!}\right) \text {, }
$$

where $D:=\frac{1}{2 \pi i} \frac{\partial}{\partial \tau}$. One obviously cannot define $J_{\kappa}$ in a similar way for $\kappa$ half-integral. However, the authors [4] found an appropriate definition of $J_{\kappa} \subseteq \mathbb{S}_{\kappa}^{!}$which makes use of so-called Zagier lifts $[8,15]$, whose main properties where discovered by Duke and Jenkins [8].

The subspaces $J_{\kappa}$ in the integral and half-integral weight cases both parallel each other and yet also exhibit a very different behavior. Recall that by the classical multiplicity one theorems, there are one-dimensional Hecke eigenspaces $F_{\kappa, j}$ and $\mathbb{F}_{k+1 / 2, j}$ for which

$$
S_{2 k}=\bigoplus_{j=1}^{t} F_{2 k, j} \quad \text { and } \quad \mathbb{S}_{k+\frac{1}{2}}=\bigoplus_{j=1}^{t} \mathbb{F}_{k+\frac{1}{2}, j} .
$$

The quotient spaces $S_{2 k}^{!} / J_{2 k}$ and $\mathbb{S}_{k+1 / 2}^{!} / J_{k+1 / 2}$ also decompose into Hecke eigenspaces

$$
S_{2 k}^{!} / J_{2 k}=\bigoplus_{j=1}^{t} E_{2 k, j} \quad \text { and } \quad \mathbb{S}_{k+\frac{1}{2}}^{!} / J_{k+\frac{1}{2}}=\bigoplus_{j=1}^{t} \mathbb{E}_{k+\frac{1}{2}, j^{\prime}}
$$

In both cases, classical cuspidal Hecke eigenforms are also weakly holomorphic Hecke eigenforms, and the eigenvalues associated to the eigenspaces $E_{2 k, j}, \mathbb{E}_{k+1 / 2, j}, F_{2 k, j}$, and $\mathbb{F}_{k+1 / 2, j}$ all match.

However, there is a substantial difference between half-integral and integral weight: while all of the eigenspaces $E_{2 k, j}$ are two-dimensional, the eigenspaces $\mathbb{E}_{k+1 / 2, j}$ are infinitedimensional [4].

In order to state our main theorem, we need some notation. We take $p$-adic limits using the framework considered in [11], which is recalled in Section 2. In particular, we take limits of formal Laurent series with coefficients in the $p$-adic completion $\widehat{\mathbb{Q}}_{p}$ of an algebraic closure of $\mathbb{Q}_{p}$. Our main result $p$-adically links each weakly holomorphic Hecke eigenform $g$ with the corresponding holomorphic Hecke eigenform $f$ with the same Hecke eigenvalues as $g$ via repeated iteration of the operator $U_{p}$. To describe the connection more precisely, for each such $f$ we build a closely related form $\widehat{f}$, using the operator $V_{p}$ and twists by the character $\chi_{p, k}(n):=\left(\frac{(-1)^{k} n}{p}\right)$. We write the Hecke eigenvalue $\lambda_{p}$ under $T_{p^{v}}$ as $\lambda_{p}=\beta+\beta^{\prime}$, where $\beta$ and $\beta^{\prime}$ are the roots of the Hecke polynomial

$$
X^{2}-\lambda_{p} X+p^{2 k-1}=(X-\beta)\left(X-\beta^{\prime}\right),
$$


chosen so that

$$
\operatorname{ord}_{p}(\beta) \leq \operatorname{ord}_{p}\left(\beta^{\prime}\right) .
$$

We then define

$$
\widehat{f}:= \begin{cases}f-\beta^{\prime} f \mid V_{p} & \text { if } f \in S_{2 k}, \\ f-\beta^{\prime} p^{-k} f \otimes \chi_{p, k}-\beta^{\prime} f \mid V_{p^{2}} & \text { if } f \in \mathbb{S}_{k+\frac{1}{2}} .\end{cases}
$$

This paper is devoted to the proof of the half-integral weight case of the following theorem.

Theorem 1.1. Suppose that $\kappa>6$ is an even integer $2 k$ or a half-integer $k+1 / 2$ and that $g$ is a weight $\kappa$ weakly holomorphic Hecke eigenform with algebraic Fourier coefficients. Furthermore, let $p \neq 2$ be a prime for which the eigenvalue $\lambda_{p}=\beta+\beta^{\prime}$ of $g$ under $T_{p^{v}}$ satisfies ( $v=1$ if $\kappa=2 k$ and $v=2$ if $\kappa=k+1 / 2$ )

$$
\operatorname{ord}_{p}(\beta)<k-1 \text {. }
$$

Then there exists $\alpha \in \widehat{\overline{\mathbb{Q}}}_{p}$ such that (as a p-adic limit)

$$
\lim _{n \rightarrow \infty} \beta^{-n} g \mid U_{p^{v n}}=\alpha \widehat{f},
$$

where $f$ is a weight $\kappa$ cuspidal Hecke eigenform with algebraic coefficients and the same Hecke eigenvalues as $g$.

Remarks.

(1) For every half-integral weight (cuspidal) Hecke eigenform $f \in \mathbb{S}_{\kappa}$, there exists (a non-cuspidal) $g \in \mathbb{S}_{\kappa}^{!}$with algebraic coefficients and the same Hecke eigenvalues as $f$, see Lemma 2.3 (1).

(2) Note that the assumption $\kappa>6$ in the theorem is not a real restriction, since otherwise there exist no cusp forms.

(3) As pointed out by the referee, in the absence of holomorphic cusp forms $\left(k<6\right.$ ), an obvious modification of the theorem (with $\widehat{f}=0$ and arbitrary $\lambda_{p}$ ) remains true for $k \geq 2$. This follows from (3.4) and was essentially proven by Duke and Jenkins [8].

In the case of integral weight, Theorem 1.1 is a special case of results proven in [11]. Our proof however differs substantially from that given in the case of integral weight. In particular, the more complicated structure of half-integral weight weakly holomorphic Hecke eigenforms requires a careful analysis where condition (1.3) becomes crucial, while the argument in integral weight is more straightforward and no analogue of condition (1.3) is needed. We do not know examples for which (1.3) does not hold true. In particular, if the famous Lehmer conjecture [14] is true, then (1.3) holds for every prime $p$ in the case $k=6$. This fact along with the surprisingly important role that the condition (1.3) plays in our proof allows us to suggest that (1.3) should not merely be considered a technical condition, but is quite interesting and, possibly, deep on its own. Furthermore, as pointed out to the authors by Kevin Buzzard, it follows from [2] (and computer calculations in [9]) that a typically much stronger inequality $\operatorname{ord}_{p}(\beta)<\frac{2 k-2}{p-1}$ is true for all primes $2<p<$ 100 other than possibly $p=59$ and $p=79$. We hence dare to state (1.3) as a conjecture. 
Conjecture 1.2. For a prime $p \geq 3$ and an integer $k \geq 6$, let $\lambda_{p}=\beta+\beta^{\prime}$ be an eigenvalue of the Hecke operator $T_{p}$ acting on $S_{2 k}$. Then

$$
\operatorname{ord}_{p}(\beta)<k-1 \text {. }
$$

We finally illustrate the congruences in Theorem 1.1 with a numerical example.

Example. The one-dimensional space $\mathbb{S}_{13 / 2}$ is generated by the function $\left(q:=e^{2 \pi i \tau}\right.$ with $\tau \in \mathbb{H}$ throughout)

$$
\begin{aligned}
\delta(\tau) & :=\frac{1}{12}\left(E_{6}(4 \tau) \theta(\tau)-120 E_{4}(4 \tau) C_{\frac{5}{2}}(\tau)\right) \\
& =q-56 q^{4}+120 q^{5}-240 q^{8}+9 q^{9}+1440 q^{12}-1320 q^{13}-704 q^{16}-\ldots,
\end{aligned}
$$

where $E_{k}$ is the weight $k$ normalized Eisenstein series, $\theta(\tau):=\sum_{n \in \mathbb{Z}} q^{n^{2}}$, and $C_{5 / 2}$ is the weight $5 / 2$ Cohen-Eisenstein series [6]. In order to $p$-adically investigate $\delta$, we use a computer to extend the large table of the $q$-expansion coefficients of $\delta$ given in [13].

We let $\Delta:=\left(E_{4}^{3}-E_{6}^{2}\right) / 1728$, and consider the weakly holomorphic cusp form $g \in \mathbb{S}_{13 / 2}^{!}$ defined by

$$
\begin{aligned}
g(\tau):= & \frac{E_{6}^{3}(4 \tau) \theta(\tau)-120 E_{6}^{2}(4 \tau) E_{4}(4 \tau) C_{\frac{5}{2}}(\tau)}{12 \Delta(4 \tau)}+6720 E_{4}(4 \tau) C_{\frac{5}{2}}(\tau) \\
= & q^{-3}-1424 q+64384 q^{4}-58275 q^{5}-11614464 q^{8}+43240944 q^{9} \\
& -1262037504 q^{12}+3433679046 q^{13}-53318226944 q^{16} \\
& +123834283776 q^{17}+\ldots
\end{aligned}
$$

For $p=3$, we have

$$
\beta=3^{2}+3^{5}+2 \cdot 3^{9}+2 \cdot 3^{10}+2 \cdot 3^{11}+O\left(3^{15}\right), \quad \beta^{\prime}=3^{9}+2 \cdot 3^{12}+2 \cdot 3^{13}+2 \cdot 3^{14}+O\left(3^{15}\right) .
$$

It is then easy to calculate that $\widehat{\delta}(q)$ is congruent modulo $3^{15}$ to

$$
-18980 q+1062880 q^{4}+2277840 q^{5}-4555680 q^{8}-13837140 q^{9}+\ldots,
$$

and observe that the first several dozen terms of the series satisfy the congruences

$$
\frac{1}{\beta} g \mid U_{9} \equiv-\frac{1801706}{404914575} \widehat{\delta}\left(\bmod 3^{3}\right)
$$

and

$$
\frac{1}{\beta^{2}} g \mid U_{81} \equiv-\frac{6896458715112926579653}{11517795085875} \widehat{\delta}\left(\bmod 3^{13}\right) .
$$

Combining this with the congruence

$$
\frac{1801706}{404914575} \equiv \frac{6896458715112926579653}{11517795085875}\left(\bmod 3^{2}\right)
$$

illustrates Theorem 1.1.

The paper is organized as follows. In Section 2, we introduce the $p$-adic framework upon which we consider formal Laurent series. After that, we briefly recall some results from $[4,8]$ about weakly holomorphic Hecke eigenforms reformatted in a manner convenient for our use. We then prove Theorem 1.1 in Section 3. 


\section{Preliminaries}

\subsection{Formal Laurent series and $p$-adic numbers}

In order to give the necessary formal structure to define all of the notation used in Theorem 1.1, we require a $p$-adic framework upon which we may state congruences of formal Laurent series. Let $p>2$ be a prime and fix an algebraic closure $\overline{\mathbb{Q}}_{p}$ of $\mathbb{Q}_{p}$ along with an embedding $\iota: \overline{\mathbb{Q}} \hookrightarrow \overline{\mathbb{Q}}_{p}$. We let $\widehat{\mathbb{Q}}_{p}$ denote the $p$-adic completion of $\overline{\mathbb{Q}}_{p}$ and normalize the $p$-adic order so that $\operatorname{ord}_{p}(p)=1$. We do not distinguish between algebraic numbers and their images under $\iota$. In particular, for algebraic numbers $a, b \in \overline{\mathbb{Q}}$ we write $a \equiv b\left(\bmod p^{m}\right)$ if $\operatorname{ord}_{p}(\iota(a-b)) \geq m$. For a formal Laurent series $H(q)=$ $\sum_{n \gg-\infty} a(n) q^{n} \in \widehat{\overline{\mathbb{Q}}}_{p}((q))$ (i.e., $q^{\ell} H \in \widehat{\overline{\mathbb{Q}}}_{p} \llbracket q \rrbracket$ for some $\left.\ell \in \mathbb{N}_{0}\right)$, we write $H \equiv 0\left(\bmod p^{m}\right)$ if $\inf _{n \in \mathbb{Z}}\left(\operatorname{ord}_{p}(a(n))\right) \geq m$.

We further require the standard operators $U_{p}, V_{p}$, and $\chi_{p, k}$ from the theory of modular forms, whose actions on formal Laurent series are given by

$$
\begin{aligned}
& \sum_{n \gg-\infty} a(n) q^{n}\left|U_{p}=\sum_{n \gg-\infty} a(p n) q^{n}, \quad \sum_{n \gg-\infty} a(n) q^{n}\right| V_{p}=\sum_{n \gg-\infty} a(n) q^{p n}, \\
& \sum_{n \gg-\infty} a(n) q^{n} \otimes \chi_{p, k}=\sum_{n \gg-\infty}\left(\frac{(-1)^{k} n}{p}\right) a(n) q^{n} .
\end{aligned}
$$

The half-integral weight Hecke operator is then defined by

$$
T_{p^{2}}=T_{p^{2}, k}:=U_{p^{2}}+p^{k-1} \chi_{p, k}+p^{2 k-1} V_{p^{2}}
$$

Here and throughout we use the convention $U_{p^{2}}=U_{p}^{2}$ and $V_{p^{2}}=V_{p}^{2}$. The Hecke operators for higher powers of $p$ satisfy the recursive relation

$$
T_{p^{2 \ell+2}}=T_{p^{2}} T_{p^{2 \ell}}-p^{2 k-1} T_{p^{2 \ell-2}} \text {. }
$$

Noting that the Hecke operators are multiplicative, the following lemma follows immediately.

Lemma 2.1. If $M, n \in \mathbb{N}$ and $F$ and $G$ are two formal Laurent series with algebraic coefficients satisfying $F \equiv G\left(\bmod p^{M}\right)$, then $F\left|T_{n^{2}} \equiv G\right| T_{n^{2}}\left(\bmod p^{M}\right)$.

The operators $U_{p}, V_{p}, \chi_{p, k}$, and $T_{p^{2}}$ furthermore map modular forms to modular forms (of possibly different level), although we do not require this in the paper. Having defined the Hecke operators, the notion of half-integral weight weakly holomorphic Hecke eigenforms is then given by (1.1), where $J_{k+1 / 2}$ is defined in the next section.

\subsection{Weakly holomorphic Hecke eigenforms}

In this section, we define the subspace $J_{k+1 / 2} \subset \mathbb{S}_{k+1 / 2}$ using Zagier lifts. These lifts were introduced by Duke and Jenkins in [8], and further (independently) studied by Alfes [1] and the authors [4] in the framework of harmonic weak Maass forms. We recall some properties of the lifts which both guarantee that our definition of weakly holomorphic Hecke eigenforms (1.1) makes sense and also play an important role in our proof of Theorem 1.1 in the next section. 
Recall that the space $\mathbb{S}_{k+1 / 2}^{!}$consists of those weakly holomorphic cusp forms $f$ whose Fourier expansions satisfy

$$
f(\tau)=\sum_{\substack{n \gg-\infty \\(-1)^{k} n \equiv 0,1(\bmod 4)}} a(n) q^{n}
$$

For each fundamental discriminant $D$ such that $(-1)^{k} D<0$, the $D$ th Zagier lift $\mathfrak{Z}_{D}$ is a linear map $\mathfrak{Z}_{D}: S_{2-2 k}^{!} \rightarrow \mathbb{S}_{k+1 / 2}^{!}$. Explicitly, if $f(\tau)=\sum_{n \gg-\infty} a(n) q^{n}$, then

$$
\mathfrak{Z}_{D}(f)(\tau):=\sum_{m>0} a(-m) m^{2 k-1} \sum_{n \mid m} \chi_{D}(n) n^{-k} q^{-\left(\frac{m}{n}\right)^{2}|D|}+\sum_{d: d D<0} t_{f}(d, D) q^{|d|}
$$

where $\chi_{D}(\cdot):=\left(\frac{D}{.}\right)$. Here the sum over $d$ runs through all discriminants for which $(-1)^{k} d>0$ and, by Lemma 2 of $[8], t_{f}(d, D)$ satisfy

$$
t_{f}\left(d m^{2}, D\right)=-m^{2 k-1} \sum_{a \mid m} \mu(a) \chi_{d m^{2}}(a) \sum_{b \mid \frac{m}{a}} \chi_{D}(b)(a b)^{-k} t_{f}\left(\left(\frac{m}{a b}\right)^{2} D, d\right),
$$

where $\mu$ is the Möbius function.

Remark. The quantities $t_{f}(d, D)$, denoted by $\operatorname{Tr}_{d, D}^{*}(f)$ in [8], are very interesting since they can be interpreted as traces of a certain function [8], and, simultaneously, as certain cycle integrals [4] whose connection with weak Maass forms was extensively studied by Duke, Imamoğlu, and Tóth in [7]. However, we do not need and do not discuss these facts in this paper.

We collect useful (known) properties of the Zagier lifts in Theorem 2.2 below. In addition to their application in our proof of Theorem 1.1, these properties illustrate the parallelism between $J_{2 k}=D^{k-1}\left(S_{2-2 k}^{!}\right)$and, following [4],

$$
J_{k+\frac{1}{2}}:=\operatorname{span}_{\mathbb{C}}\left\{\mathfrak{Z}_{D}(f) \mid f \in S_{2-2 k}^{!}, D \text { fundamental with }(-1)^{k} D<0\right\} \subseteq \mathbb{S}_{k+\frac{1}{2}}^{!} .
$$

\section{Theorem 2.2.}

(1) The space $J_{k+1 / 2}$ is stable under the action of the Hecke algebra.

(2) We have $J_{k+1 / 2} \cap \mathbb{S}_{k+1 / 2}=\{0\}$.

(3) We have an isomorphism between $\mathbb{S}_{k+1 / 2}^{!} / J_{k+1 / 2}$ and infinitely many copies of $\mathbb{S}_{k+1 / 2}$ as modules over the Hecke algebra.

(4) Iff $\in S_{2-2 k}^{!}$has integral Fourier coefficients, then so does $\mathfrak{Z}_{D}(f)$.

Proof. Theorem 2.2 (1) follows by Lemma 2 of [8], Theorem 2.2 (2) is implied by an orthogonality condition in Proposition 6.3 of [4], Theorem 2.2 (3) is implied by Theorem 1.6 of [4], and Theorem $2.2(4)$ is Theorem 1 of [8].

By Theorem $2.2(2), F \in J_{k+1 / 2}$ is entirely determined by its principal part $P_{F}(q):=$ $\sum_{n<0} a(n) q^{n}$. Noting that $R_{F}:=F-P_{F}$ is bounded towards $i \infty$, we obtain a natural decomposition

$$
F=P_{F}+R_{F}
$$


Theorem 2.2 (2) also immediately implies that every holomorphic Hecke eigenform is a weakly holomorphic Hecke eigenform. Furthermore, Theorem 2.2 (1) allows one to define the action of Hecke operators on the quotient space $\mathbb{S}_{k+1 / 2} / J_{k+1 / 2}$, and therefore to justify the definition (1.1) of weakly holomorphic Hecke eigenforms for half-integral weight.

Theorem 2.2 (3) explains the decomposition (1.2) in the half-integral weight case, while the integral weight decomposition follows by Theorem 1 of [10] and its refinement, Theorem 1.2 of [5]. However, since we are interested in $p$-adic analysis, we require a slightly stronger splitting where we may take the representatives with algebraic coefficients. A standard linear algebra argument which is well-known to experts implies the following lemma. We provide a detailed proof for the reader's convenience.

\section{Lemma 2.3 .}

(1) Every space $\mathbb{E}_{k+1 / 2, j}$ in decomposition (1.2) has a basis consisting of (infinitely many) cosets whose representatives are weakly holomorphic Hecke eigenforms with algebraic Fourier coefficients.

(2) If $g \in \mathbb{S}_{k+1 / 2}^{!}$has algebraic coefficients, then, for each $1 \leq j \leq t$, there exists a representative of its projection into $\mathbb{E}_{k+1 / 2, j}$ with algebraic coefficients.

Remark. The (totally real) algebraic number field in (1) is generated over $\mathbb{Q}$ by all of the eigenvalues of weight $2 k$ cusp forms and hence only depends on $k \geq 6$.

Proof.

(1) Since $\mathbb{S}_{k+1 / 2}^{!}$has a basis consisting of functions with rational Fourier coefficients by Proposition 1 of [8], (1) follows immediately from (2).

(2) Recall that there exists $N \in \mathbb{N}$ such that the integral basis $f_{k+1 / 2, m}$ of $\mathbb{S}_{k+1 / 2}^{!}$in (10) of [8] satisfies

$$
f_{k+\frac{1}{2}, m}(\tau)=q^{m}+O\left(q^{N}\right)
$$

with $m<N$. Comparing the coefficient in front of $q^{m}$, we see immediately that any $g=\sum_{m<N} a_{m} f_{k+1 / 2, m} \in \mathbb{S}_{k+1 / 2}^{!}$has algebraic coefficients if and only if each $a_{m}$ is algebraic. Thus it is enough to show part (2) for each $f_{k+1 / 2, m}$. In particular we may assume that $g$ has rational coefficients. We next decompose $g$ as $g=\sum_{j=1}^{t} g_{j}$ with $g_{j} \in \mathbb{E}_{k+1 / 2, j}$. Note that $g_{j}$ is only uniquely determined up to an element of $J_{k+1 / 2}$.

Since the eigenvalues of weakly holomorphic modular forms are the same as those of classical cusp forms by Theorem 2.2 (3), an element of $\mathbb{S}_{k+1 / 2}^{!}$is a Hecke eigenform if and only if it is an eigenfunction for finitely many Hecke operators $T_{n_{1}}, \ldots, T_{n_{d}}$. For each $1 \leq j \leq t$ and $1 \leq r \leq d$, we denote the eigenvalues corresponding to $\mathbb{E}_{k+1 / 2, j}$ under $T_{n_{r}}$ by $\alpha_{n_{r}, j}$. We now fix $1 \leq j_{0} \leq t$ and project $g$ into $\mathbb{E}_{k+1 / 2, j_{0}}$. Since $J_{k+1 / 2}$ is Hecke-stable, one inductively concludes that there exists $R_{j_{0}} \in J_{k+1 / 2}$ such that

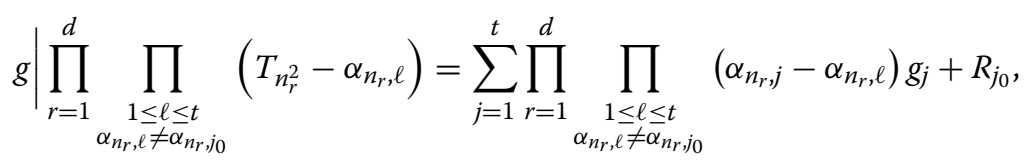


where $\alpha_{n, j}$ denotes the eigenvalue of $g_{j}$ under $T_{n_{j}^{2}}$. If $j \neq j_{0}$, then multiplicity one implies that $\alpha_{n_{r}, \ell} \neq \alpha_{n_{r}, j_{0}}$ for some $r$ and hence the constant in front of $g_{j}$ in (2.7) is zero. Therefore the sum in (2.7) reduces to $j=j_{0}$ and, by Theorem $2.2(1)$, we obtain

$$
g_{j_{0}}=\frac{1}{\lambda_{j_{0}}} g \mid \prod_{r=1}^{d} \prod_{\substack{1 \leq \ell \leq t \\ \alpha_{n_{r}, \ell \neq \alpha_{n_{r}, j_{0}}}}}\left(T_{n_{r}^{2}}-\alpha_{n_{r}, \ell}\right)-\frac{1}{\lambda_{j_{0}}} R_{j_{0}},
$$

with

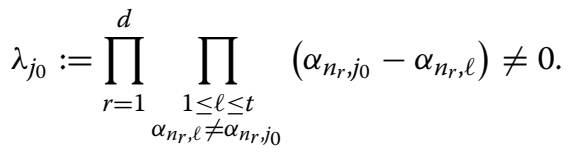

Since $g$ has rational coefficients and rationality is preserved by the Hecke operators, the first term on the right-hand side of (2.8) is in the number field generated by all of the eigenvalues of the Hecke operators. At the same time, it is in the same coset as $g_{j_{0}}$, completing the proof, since $R_{j_{0}}$, and thus the second summand in (2.8), is in $J_{k+1 / 2}$.

\section{Proof of Theorem 1.1}

In this section, we prove Theorem 1.1 for half-integral weight.

\subsection{Congruences relating $U_{p^{2}}$ and $T_{p^{2}}$}

The following lemma allows us to $p$-adically relate the iterated action of $U_{p^{2}}$ and $T_{p^{2}}$.

Lemma 3.1. Let $p$ be a prime, $A, k \in \mathbb{N}$, and $h \in \overline{\mathbb{Q}}((q))$ satisfy

$$
h \mid U_{p^{2}}^{n} \equiv 0 \quad\left(\bmod p^{(k-1)(n-A)}\right)
$$

for every $n \in \mathbb{N}_{0}$. Then we also have

$$
h \mid T_{p^{2}, k}^{n} \equiv 0 \quad\left(\bmod p^{(k-1)(n-A)}\right) .
$$

Proof. We use definition (2.1) and expand $\left(U_{p^{2}}+p^{k-1} \chi_{p, k}+p^{2 k-1} V_{p^{2}}\right)^{n}$. Denoting by $a, b$, and $c$ the total powers of $U_{p^{2}}, \chi_{p, k}$, and $V_{p^{2}}$, respectively in a single term, we have $a+b+c=n$. We claim that every individual term satisfies the required congruence. We first note that

$$
\chi_{p, k} U_{p^{2}}=0=V_{p^{2}} \chi_{p, k}
$$

since acting by $\chi_{p, k}$ annihilates coefficients that are divisible by $p$. Furthermore, $V_{p^{2}} U_{p^{2}}$ is the identity. Proceeding by induction on the number of different adjacent operators, we conclude that all non-vanishing terms are of the form

$$
p^{(2 k-1) c+(k-1) b} h\left|U_{p^{2}}^{a-r} \otimes \chi_{p, k}^{b}\right| V_{p^{2}}^{c-r},
$$

where $r$ denotes the number of times that $V_{p^{2}} U_{p^{2}}$ is replaced with the identity. Since $r \leq \min (a, c)$, the $p$-adic order of each term is, by (3.1), at least

$$
(2 k-1) c+(k-1) b+(k-1)(a-c-A)=(k-1)(n-A)+c \geq(k-1)(n-A),
$$

as claimed. 


\subsection{The action of $U_{p^{2}}$}

Our next proposition demonstrates an important similarity between $J_{2 k}$ and $J_{k+1 / 2}$ : elements of both spaces are $p$-adically annihilated by repeated application of $U_{p^{2}}$.

Proposition 3.2. If $f \in J_{k+1 / 2}$ has algebraic Fourier coefficients, then there exists a constant $M \in \mathbb{N}_{0}$ such that, for every $n \in \mathbb{N}_{0}$,

$$
f \mid U_{p^{2}}^{n} \equiv 0\left(\bmod p^{(k-1) n-M}\right)
$$

Proof. In Proposition 1 of [8], Duke and Jenkins constructed a basis of $M_{2-2 k}^{!}$which consists of forms with integral Fourier coefficients. After subtracting a constant multiple of such a form in $M_{2-2 k}^{!}$whose constant coefficient is positive and minimal, one can construct a basis for $S_{2-2 k}^{!}$with integral coefficients. Since $f \in J_{k+1 / 2}$ has algebraic coefficients, there exist discriminants $D_{1}, \ldots D_{r}$, basis elements $f_{1}, \ldots, f_{\ell} \in S_{2-2 k}^{!}$, and $\gamma_{v, j} \in \mathbb{C}$ for which

$$
f=\sum_{\nu=1}^{r} \sum_{j=1}^{\ell} \gamma_{\nu, j} \mathfrak{Z}_{D_{v}}\left(f_{j}\right) .
$$

By comparing principal parts on both sides using (2.2), we furthermore conclude that $\gamma_{v, j} \in \overline{\mathbb{Q}}$. It thus suffices to prove the required congruence for every individual term of the sum. Since repeated application of $U_{p^{2}}$ eliminates their (finite) principal parts, we may ignore these principal parts by choosing $M$ large. Furthermore, integrality of $\gamma_{\nu, j}$ may be assumed by choosing an appropriate $M$. By the decomposition (2.5), it suffices to show that for every $f$ with integer coefficients and a fundamental discriminant $D$

$$
R_{\mathfrak{Z}_{D}(f)} \mid U_{p^{2}}^{n} \equiv 0\left(\bmod p^{(k-1) n}\right) .
$$

We remark that Duke and Jenkins essentially proved (3.4) while showing integrality of $R_{\mathfrak{Z}_{D}}(f)$ in Theorem 1 of [8]. To finish the proof of (3.3), note that by Theorem 2.2 (4), $R_{\mathfrak{Z}_{D}(f)}$ has integer coefficients given by (2.2) and thus the congruence follows, since in (2.3) $a b$ is a divisor of $m$ and $m$ is divisible by $p^{n}$.

We next prove that the $p$-adic limit in Theorem 1.1 exists.

Proposition 3.3. Let $g \in \mathbb{S}_{k+1 / 2}^{!}$be a Hecke eigenform with algebraic Fourier coefficients. If $\operatorname{ord}_{p}(\beta)<k-1$, then the $p$-adic limit $\lim _{n \rightarrow \infty} \beta^{-n} g \mid U_{p^{2}}^{n}$ exists.

Proof. If $g \in \mathbb{S}_{k+1 / 2}^{!}$is a Hecke eigenform with eigenvalue $\lambda_{p}=\beta+\beta^{\prime}$ under $T_{p^{2}}$, then, by (1.1) and (2.1), there exists $r \in J_{k+1 / 2}$ with algebraic coefficients such that

$$
g\left|U_{p^{2}}+p^{k-1} g \otimes \chi_{p, k}+p^{2 k-1} g\right| V_{p^{2}}=\left(\beta+\beta^{\prime}\right) g+r .
$$

Let

$$
g_{\beta}:=g-\beta p^{-k} g \otimes \chi_{p, k}-\beta g \mid V_{p^{2}} \quad \text { and } \quad g_{\beta^{\prime}}:=g-\beta^{\prime} p^{-k} g \otimes \chi_{p, k}-\beta^{\prime} g \mid V_{p^{2}} .
$$

Equation (3.2) together with the fact that $V_{p^{2}} U_{p^{2}}$ is the identity and $\beta \beta^{\prime}=p^{2 k-1}$ imply that

$$
g_{\beta} \mid U_{p^{2}}=\beta^{\prime} g_{\beta}+r \quad \text { and } \quad g_{\beta^{\prime}} \mid U_{p^{2}}=\beta g_{\beta^{\prime}}+r .
$$


We now apply $\beta^{-1} U_{p^{2}} n$ times to the identity

$$
\left(\beta-\beta^{\prime}\right) g=\beta g_{\beta^{\prime}}-\beta^{\prime} g_{\beta} \text {. }
$$

By (3.6), we obtain inductively

$$
\beta^{-n}\left(\beta-\beta^{\prime}\right) g\left|U_{p^{2}}^{n}=\beta g_{\beta^{\prime}}-\beta^{\prime}\left(\frac{\beta^{\prime}}{\beta}\right)^{n} g_{\beta}+\sum_{m=0}^{n-1} \frac{\beta^{n-m}-\beta^{\prime n-m}}{\beta^{n}} r\right| U_{p^{2}}^{m} .
$$

From $\operatorname{ord}_{p}\left(\beta^{\prime}\right)>\operatorname{ord}_{p}(\beta)$, it follows that

$$
\lim _{n \rightarrow \infty} \beta^{\prime}\left(\frac{\beta^{\prime}}{\beta}\right)^{n} g_{\beta}=0 .
$$

Since

$$
\operatorname{ord}_{p}\left(\frac{\beta^{\prime n-m}}{\beta^{n}}\right)>\operatorname{ord}_{p}\left(\beta^{-m}\right),
$$

it thus suffices to check $p$-adic convergence of the sum

$$
\sum_{m=0}^{\infty} \beta^{-m} r \mid U_{p^{2}}^{m}
$$

From the assumption that $\operatorname{ord}_{p}(\beta)<k-1$, this however follows by Proposition 3.2.

We next prove a special case of Theorem 1.1 for $g=f \in \mathbb{S}_{k+1 / 2}$. In this case the condition $\operatorname{ord}_{p}(\beta)<k-1$ can be relaxed to $\operatorname{ord}_{p}(\beta)<k-1 / 2$ (equivalently, $\left.\operatorname{ord}_{p}(\beta)<\operatorname{ord}_{p}\left(\beta^{\prime}\right)\right)$.

Proposition 3.4. If $\in \in \mathbb{S}_{k+1 / 2}$ is a Hecke eigenform with algebraic Fourier coefficients for which $\operatorname{ord}_{p}(\beta)<\operatorname{ord}_{p}\left(\beta^{\prime}\right)$, then $f$ satisfies (1.4) with $\alpha=\frac{\beta}{\beta-\beta^{\prime}}$.

Proof. Note that for $f \in \mathbb{S}_{k+1 / 2}$, we have $r=0$ in (3.5). Thus (3.7) reads

$$
\beta^{-n}\left(\beta-\beta^{\prime}\right) f \mid U_{p^{2}}^{n}=\beta f_{\beta^{\prime}}-\beta^{\prime}\left(\frac{\beta^{\prime}}{\beta}\right)^{n} f_{\beta},
$$

and (3.8) implies the desired result.

\subsection{The action of $T_{p^{2}}$}

We next consider repeated action of the Hecke operators $T_{p^{2}}$ on $\mathbb{S}_{k+1 / 2}^{!}$.

Proposition 3.5. Suppose that $g \in \mathbb{S}_{k+1 / 2}^{!}$has algebraic Fourier coefficients. Then there exists an integer $L$ such that for every integer $\ell>L$ there exists a holomorphic modular form $G_{\ell} \in \mathbb{S}_{k+1 / 2}$ with algebraic Fourier coefficients for which

$$
g \mid T_{p^{2}}^{\ell} \equiv G_{\ell} \quad\left(\bmod p^{(k-1)(\ell-L)}\right) .
$$

Proof. We decompose $g$ as in (2.5). Since repeated action of $U_{p^{2}}$ eliminates $P_{g}$, Lemma 3.1 implies the existence of an integer $L$ such that

$$
P_{g} \mid T_{p^{2}}^{\ell} \equiv 0 \quad\left(\bmod p^{(k-1)(\ell-L)}\right) .
$$

Since the basis elements (2.6) have integral coefficients, a linear combination which has the same principal part as $P_{g} \mid T_{p^{2}}^{\ell}$ vanishes modulo $p^{(k-1)(\ell-L)}$. Subtracting this linear 
combination from $g \mid T_{p^{2}}^{\ell}$ yields a cusp form $G_{\ell}$ which satisfies the conditions given in the proposition.

For the repeated action of the Hecke operators on elements of $J_{k+1 / 2}$, we require a more precise statement.

Proposition 3.6. If $g \in J_{k+1 / 2}$ has algebraic Fourier coefficients, then there exists an $L$ such that for every $\ell \in \mathbb{N}_{0}$, we have

$$
g \mid T_{p^{2}}^{\ell} \equiv 0\left(\bmod p^{(k-1)(\ell-L)}\right) .
$$

Proof. By Proposition 3.2, there exists $L$ such that for all $\ell \in \mathbb{N}_{0}$, we have

$$
g \mid U_{p^{2}}^{\ell} \equiv 0 \quad\left(\bmod p^{(k-1)(\ell-L)}\right) .
$$

Lemma 3.1 then immediately implies the proposition.

\subsection{Proof of Theorem 1.1}

We are now ready to prove Theorem 1.1.

Proof of Theorem 1.1. Since $g \in \mathbb{S}_{k+1 / 2}^{!}$is a weakly holomorphic Hecke eigenform (with algebraic coefficients), there exists $r \in J_{k+1 / 2}$ with algebraic Fourier coefficients such that

$$
g \mid T_{p^{2}}=\lambda_{p} g+r
$$

Repeatedly acting with $T_{p^{2}}$, we obtain

$$
g\left|T_{p^{2}}^{\ell}=\lambda_{p}^{\ell} g+\sum_{m=1}^{\ell} \lambda_{p}^{\ell-m} r\right| T_{p^{2}}^{m-1}
$$

By Proposition 3.5, there exist $G_{\ell} \in \mathbb{S}_{k+1 / 2}$ and $L_{g} \in \mathbb{Z}$ such that

$$
G_{\ell} \equiv \lambda_{p}^{\ell} g+\sum_{m=1}^{\ell} \lambda_{p}^{\ell-m} r \mid T_{p^{2}}^{m-1}\left(\bmod p^{(k-1)\left(\ell-L_{g}\right)}\right) .
$$

We abbreviate

$$
\mu:=\operatorname{ord}_{p}\left(\lambda_{p}\right)=\operatorname{ord}_{p}(\beta)<k-1
$$

and choose $L_{r}$ to satisfy Proposition 3.6 for $r$. Thus we obtain that

$$
\lambda_{p}^{-\ell} G_{\ell} \equiv g+p^{-(k-1)\left(L_{r}+1\right)} r_{\ell}\left(\bmod p^{(k-1)\left(\ell-L_{g}\right)-\mu \ell}\right),
$$

where

$$
r_{\ell}:=p^{(k-1)\left(L_{r}+1\right)} \lambda_{p}^{-\ell} \sum_{m=1}^{\ell} \lambda_{p}^{\ell-m} r \mid T_{p^{2}}^{m-1} \in J_{k+\frac{1}{2}}
$$

has $p$-integral Fourier coefficients. This follows by (3.9) and Proposition 3.6, since

$$
\lambda_{p}^{\ell-m} r \mid T_{p^{2}}^{m-1} \equiv 0\left(\bmod p^{\mu(\ell-m)+(k-1)\left(m-1-L_{r}\right)}\right) .
$$

Since $g$ is a Hecke eigenform, we have $g \in \mathbb{E}_{k+1 / 2, j_{0}}$ for some $1 \leq j_{0} \leq t$. We next apply operators to both sides of (3.10) in order to project $G_{\ell}$ into the eigenspace $\mathbb{F}_{k+1 / 2, j_{0}}$. As in the proof of Lemma 2.3 , we choose $n_{1}, \ldots, n_{d}$ so that an element of $\mathbb{S}_{k+1 / 2}^{!}$is a Hecke eigenform if and only if it is an eigenfunction under each $T_{n_{r}^{2}}$ for $r=1, \ldots, d$ with 
$\left(n_{r}, p\right)=1$. For each eigenspace $\mathbb{E}_{k+1 / 2, j}$, we denote the corresponding eigenvalue under $T_{n_{r}^{2}}$ by $\alpha_{n_{r}, j}$. Now choose $L \in \mathbb{N}_{0}$ such that

$$
\left|\lambda_{j_{0}}\right|+\sum_{r=1}^{d} \sum_{\substack{1 \leq j \leq t \\ \alpha_{n_{r}, j \neq 0}}}\left|\operatorname{ord}_{p}\left(\alpha_{n_{r}, j}\right)\right| \leq(k-1) L,
$$

with $\lambda_{j_{0}}$ as in (2.9). Then, by (3.10) and Lemma 2.1, we conclude that

$$
\lambda_{p}^{-\ell} G_{\ell, j_{0}} \equiv g_{j_{0}}+p^{-(k-1)\left(L_{r}+L+1\right)} r_{\ell, j_{0}}\left(\bmod p^{(k-1)\left(\ell-\left(L_{g}+L\right)\right)-\mu \ell}\right),
$$

where

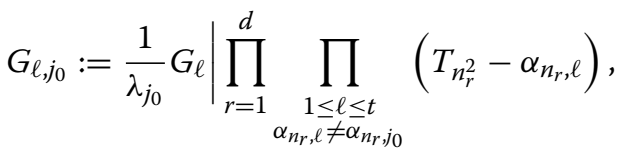

$$
\begin{aligned}
& g_{j_{0}}:=\frac{1}{\lambda_{j_{0}}} g \mid \prod_{r=1}^{d} \prod_{\substack{1 \leq \ell \leq t \\
\alpha_{n_{r}, \ell \neq \alpha_{n_{r}, j_{0}}}}}\left(T_{n_{r}^{2}}-\alpha_{n_{r}, \ell}\right), \\
& r_{\ell, j_{0}}:=\frac{p^{(k-1) L}}{\lambda_{j_{0}}} r_{\ell} \mid \prod_{r=1}^{d} \prod_{\substack{1 \leq \ell \leq t \\
\alpha_{n_{r}, \ell \neq \alpha_{n_{r}, j_{0}}}}}\left(T_{n_{r}^{2}}-\alpha_{n_{r}, \ell}\right) .
\end{aligned}
$$

Since $r_{\ell}$ has $p$-integral Fourier coefficients, (3.12) implies that $r_{\ell, j_{0}}$ also has $p$-integral Fourier coefficients, while $G_{\ell, j_{0}}$ is the projection of $G_{\ell}$ into $\mathbb{F}_{k+1 / 2, j_{0}}$ and $g_{j_{0}}$ is a representative for the projection of $g$ into $\mathbb{E}_{k+1 / 2, j_{0}}$ by (2.8). Since $G_{\ell, j_{0}}$ is a cuspidal Hecke eigenform with the same eigenvalues as $g$, we conclude, by multiplicity one, that for every $\ell \in \mathbb{N}$ there exists a constant $h(\ell) \in \overline{\mathbb{Q}}$ for which

$$
\lambda_{p}^{-\ell} G_{\ell, j_{0}}=h(\ell) f,
$$

with $f$ as in the statement of the theorem.

For each $n \in \mathbb{N}$, we then apply $\beta^{-n} U_{p^{2}}^{n}$ to (3.13) to obtain

$$
\beta^{-n} h(\ell) f\left|U_{p^{2}}^{n} \equiv \beta^{-n} g_{j_{0}}\right| U_{p^{2}}^{n}+p^{-(k-1)\left(L_{r}+L+1\right)} \beta^{-n} r_{\ell, j_{0}} \mid U_{p^{2}}^{n}\left(\bmod p^{(k-1)\left(\ell-\left(L_{g}+L\right)\right)-\mu \ell-\mu n}\right) .
$$

Note that since $k-1-\mu>0$, we have, for $\ell \gg n^{1+\varepsilon}$ and $n \rightarrow \infty$,

$$
(k-1)\left(\ell-\left(L_{g}+L\right)\right)-\mu \ell-\mu n \rightarrow \infty .
$$

Thus (3.14) with $\ell=n^{2}$ and $n$ sufficiently large holds for every $M \in \mathbb{N}$ modulo $p^{M}$. Similarly, Proposition 3.2 together with $k-1-\mu>0$ implies that the second term on the right-hand side of (3.14) vanishes modulo $p^{M}$ for $M \in \mathbb{N}$ for $n$ sufficiently large. We conclude that for every $M \in \mathbb{N}$, there exist $N \in \mathbb{N}$ and $h\left(n^{2}\right) \in \overline{\mathbb{Q}}$ such that for all $n>N$

$$
\beta^{-n} h\left(n^{2}\right) f\left|U_{p^{2}}^{n} \equiv \beta^{-n} g_{j_{0}}\right| U_{p^{2}}^{n}\left(\bmod p^{M}\right) .
$$

Now note that since $g \in \mathbb{E}_{k+1 / 2, j_{0}}$, it equals its projection into $\mathbb{E}_{k+1 / 2, j_{0}}$ and hence $g-$ $g_{j_{0}} \in J_{k+1 / 2}$. Since $g$ and $g_{j_{0}}$ both have algebraic coefficients, $g-g_{j_{0}}$ also does, and hence, by Proposition 3.2, we conclude that for $n$ sufficiently large

$$
\beta^{-n} h\left(n^{2}\right) f\left|U_{p^{2}}^{n} \equiv \beta^{-n} g_{j_{0}}\right| U_{p^{2}}^{n} \equiv \beta^{-n} g \mid U_{p^{2}}^{n}\left(\bmod p^{M}\right) .
$$


Proposition 3.4 allows us to calculate the $p$-adic $\operatorname{limit}_{n \rightarrow \infty} \beta^{-n} f \mid U_{p^{2}}^{n}$, while the righthand side of (3.15) exists by Proposition 3.3. This implies the existence of the $p$-adic limit $\lim _{n \rightarrow \infty} h\left(n^{2}\right) \in \widehat{\mathbb{Q}}_{p}$, and letting

$$
\alpha:=\frac{\beta}{\beta-\beta^{\prime}} \lim _{n \rightarrow \infty} h\left(n^{2}\right),
$$

we conclude Theorem 1.1.

\section{Acknowledgements}

The research of the first author was supported by the Alfried Krupp Prize for Young University Teachers of the Krupp Foundation and by the Deutsche Forschungsgemeinschaft (DFG) Grant No. BR 4082/3-1. The research of the second author is supported by Simons Foundation Collaboration Grant. The research of the third author was supported by grant project number 27300314 of the Research Grants Council.

The authors would like to thank Kevin Buzzard for helpful comments concerning Conjecture 1.2.

\section{Author details}

${ }^{1}$ Mathematical Institute, University of Cologne, Weyertal 86-90, 50931 Cologne, Germany. ${ }^{2}$ Department of Mathematics, University of Hawaii, Honolulu, HI 96822-2273, USA. ${ }^{3}$ Department of Mathematics, University of Hong Kong, Pokfulam, Hong Kong.

Received: 17 June 2015 Accepted: 5 October 2015

Published online: 21 December 2015

\section{References}

1. Alfes, C: Formulas for the coefficients of half-integral weight harmonic Maass forms. Math. Z. 227, 769-795 (2014)

2. Berger, L, Li, H, Zhu, H: Construction of some families of 2-dimensional crystalline representations. Math. Ann. 329, 365-377 (2004)

3. Bringmann, K, Guerzhoy, P, Kane, B: Mock modular forms as $p$-adic modular forms. Trans. Amer. Math. Soc. 364, 2393-2410 (2012)

4. Bringmann, K, Guerzhoy, P, Kane, B: Shintani lifts and fractional derivatives for harmonic weak Maass forms. Adv. Math. 255, 641-671 (2014)

5. Bringmann, K, Guerzhoy, P, Kent, Z, Ono, K: Eichler-Shimura theory for mock modular forms. Math. Ann. 355, 1085-1121 (2013)

6. Cohen, $\mathrm{H}$ : Sums involving the values at negative integers of L-functions of quadratic characters. Math. Ann. 217, 271-285 (1975)

7. Duke, W, Imamoḡlu, Ö, Tóth, Á: Cycle integrals of the $j$-function and mock modular forms. Ann. of Math. 173, 947-981 (2011)

8. Duke, W, Jenkins, P: Integral traces of singular values of weak Maass forms. Algebra and Number Theory. 2, 573-593 (2008)

9. Gouvêa, F: Where the slopes are. J. Ramanujan Math. Soc. 16, 75-99 (2001)

10. Guerzhoy, P: Hecke operators for weakly holomorphic modular forms and supersingular congruences. Proc. Amer. Math. Soc. 136, 3051-3059 (2008)

11. Guerzhoy, P, Kent, Z, Ono, K: $p$-adic coupling of mock modular forms and shadows. Proc. Natl. Acad. Sci. USA 107, 6169-6174 (2010)

12. Kohnen, W: Modular forms of half-integral weight on $\Gamma_{0}(4)$. Math. Ann. 248, 249-266 (1980)

13. Kohnen, W, Zagier, D: Values of $L$-series of modular forms at the center of the critical strip. Invent. Math. 64, 175-198 (1981)

14. Lehmer, D: The vanishing of Ramanujan's $\tau(n)$. Duke Math. J. 14, 429-433 (1947)

15. Zagier, D: Traces of Singular Moduli in Motives, polylogarithms and Hodge theory, Part I. International Press of Boston, Boston, MA, USA (2002) 